\title{
Psychokardiologie - aktuelle Leitlinien und klinische Realität
}

Christoph Herrmann-Lingen

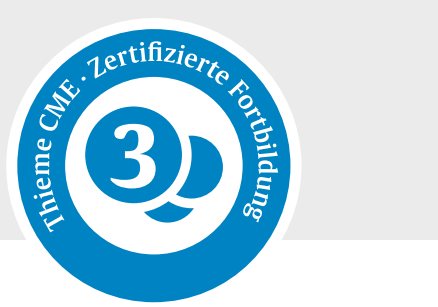

\section{Die Psychokardiologie hat in den letzten Jahrzehnten zur Bedeutung psychosozialer Faktoren für Entstehung, Verlauf und Behandlung von Herz-Kreislauf-Erkrankungen erhebliche Er- kenntnisfortschritte erbracht, die sich in aktuellen Leitlinien und Positionspapieren abbilden. Der Beitrag fasst den heutigen Wissensstand zusammen und will dafür sensibilisieren, seine vielfach noch defizitäre Umsetzung in die Versorgungspraxis zu verbessern.}

\section{FALLBEISPIEL}

\section{Teil 1}

Herr M. ist ein 45-jähriger, getrennt lebender Produktionsarbeiter. Die Ehefrau war vor 4 Jahren mit den beiden Kindern (damals 8 und 12 Jahre alt) in den benachbarten Landkreis gezogen. Seither gibt es regelmäßig Konflikte um den Unterhalt und den Umgang mit den Kindern, die Herr M. selten zu sehen bekommt und die sich zunehmend von ihm abwenden. Die finanzielle Situation ist wegen der Unterhaltszahlungen angespannt. In der seit 2 Jahren bestehenden neuen Beziehung fühlt er sich nicht wirklich wohl, da die Partnerin ( - 2 J.) erheblich durch ihren Beruf als Reinigungskraft und die pubertäre Tochter eingespannt ist. So finden Treffen meist nur an Wochenenden statt und stehen in Konkurrenz zu gelegentlichen Besuchen der Kinder.

Herr M. ist seit dem 15. Lebensjahr Raucher (aktuell ca. 30 Zigaretten pro Tag; 40 Pack Years). Er ernährt sich von Kantinenessen, Fast Food und Brot mit deftigem Aufschnitt; Obst und Gemüse isst er nur selten. Bei einer Größe von 1,79 m wiegt er $92 \mathrm{~kg}$. Seit einigen Jahren ist ein erhöhter Blutdruck bekannt (Werte zunächst um $150 / 90 \mathrm{mmHg}$, seit einer Verschlechterung des Arbeitsklimas mit vermehrtem Konkurrenzdruck bei instabiler wirtschaftlicher Lage des Unternehmens und drohenden Entlassungen teilweise Werte bis $200 / 110 \mathrm{mmHg}$ ). Blutdruckmedikamente nimmt er nur „bei Bedarf“. Herr M. hat früher Fußball gespielt, musste dies aber mit 32 Jahren nach einem Bänderriss aufgeben. Bis auf gelegentliche Unternehmungen mit den Kindern ist er sportlich nicht mehr aktiv.

Es bestehen einige Sozialkontakte in der Freiwilligen Feuerwehr, keine engen Freundschaften. Haupt-Gesprächspartnerin ist die neue Partnerin, die durch ihre eigene Belastung aber stark eingespannt ist. Herr M. tut sich ohnehin schwer, über emotionale Themen zu sprechen. Eine mutmaßlich depressive Phase nach Auszug der Ehefrau hatte er durch vermehrtes Arbeiten zu überwinden versucht. In den gegenwärtigen Konflikten am Arbeitsplatz fühlt er sich einsam („da kann mir sowieso niemand helfen“). Als er eines Tages bei der Arbeit einen drückenden Schmerz hinter dem Brustbein bekommt und einen Schwächeanfall erleidet, schickt ihn der Vorarbeiter zum Arzt. Dort sind die Beschwerden wieder abgeklungen. Im EKG und Labor (Troponin) findet sich kein Hinweis auf einen akuten Herzinfarkt. Der Arzt empfiehlt aber eine weitere kardiologische Abklärung und eine Einstellung der koronaren Risikofaktoren. Dieser Empfehlung folgt Herr M. nicht, da die Beschwerden ja wieder gebessert seien. Insgeheim hat er Sorge, durch Untersuchungen oder Behandlungen länger bei der Arbeit zu fehlen und seinen Arbeitsplatz zu verlieren. $\mathrm{Er}$ möchte sich vor den Kollegen in der momentanen Situation auch keine Blöße geben. Zudem ängstigt ihn die Vorstellung eines möglichen operativen Eingriffs. Den Gedanken, dass eine ernsthafte Herzkrankheit vorliegen könnte, schiebt er beiseite. In der Folgezeit wiederholt auftretende Thoraxschmerzen unter Belastung führt er auf Trainingsmangel und das Rauchen zurück, das er aber weiterhin als Stresspuffer benötigt und daher nicht aufgeben kann.

\section{Einleitung}

Zusammenhänge zwischen psychischem Befinden und Herzfunktionen bzw. -krankheiten sind seit der Antike bekannt. So erwähnt bereits der Papyrus Ebers aus dem 16. vorchristlichen Jahrhundert, dass Trübnis das Herz einenge, durch Zorn Finsternis in den Leib eindringe und das 
Herz „fresse“ [1]. Das Herz als gefühlter Sitz der Seele bzw. interpersoneller Verbundenheit findet sich vielfach in literarischen Metaphern und der Umgangssprache. Heute sind die Wechselbeziehungen psychosozialer und somatischer Vorgänge im Kontext von Herzbeschwerden und -erkrankungen gut belegt. Psychokardiologische Inhalte haben Eingang in Leitlinien und Positionspapiere zur Behandlung von Herz-Kreislauf-Erkrankungen und zunehmend auch in die Aus-, Fort- und Weiterbildung von Ärzten und Psychotherapeuten gefunden. Sie stellen die Grundlage für eine verstärkte Beachtung psychosozialer Aspekte in kardiologischen Präventions-, Behandlungs- und Rehabilitationsangeboten sowie für die Entwicklung spezifischer psychokardiologischer Behandlungsangebote dar.

\section{Merke}

Trotz des Vorhandenseins von Leitlinieninhalten zur Psychokardiologie sind Behandlungsangebote zu dieser Thematik vielfach erst ungenügend in der Praxis angekommen.

Der vorliegende Beitrag versucht in 2 Strängen, einerseits einen Überblick über den heutigen Wissensstand der Psychokardiologie zu geben und andererseits einen Patientenfall mit den trotz des fortgeschrittenen Wissens typischen Problemen zu skizzieren. Beide Stränge sollen abschließend zusammengeführt und verbunden werden.

\section{Psychosomatische Effekte auf das Herz-Kreislauf-System}

\section{Einflussfaktoren auf Entstehung und Verlauf ausgewählter kardiovaskulärer Erkrankungen}

Die europäische Leitlinie zur Prävention von Herz-Kreislauf-Erkrankungen [2] benennt folgende psychosoziale Risikofaktoren für kardiovaskuläre Erkrankungen:

- niedrigen sozioökonomischen Status

- mangelhafte soziale Unterstützung

- Stress im Beruf und Familienleben

- Feindseligkeit

- Depression

- Angst

- andere psychische Störungen

Insbesondere die Depression gilt als weitgehend gesicherter prognostischer Risikofaktor bei Patienten mit koronarer Herzkrankheit [3-5], Herzinsuffizienz [5, 6] und implantierten Defibrillatoren [7].

\section{Merke}

Epidemiologische Befunde belegen die Bedeutung psychischer und sozialer Faktoren für Entstehung und Verlauf von Herz-Kreislauf-Erkrankungen.
Im aktuellen Positionspapier der Deutschen Gesellschaft für Kardiologie zu psychosozialen Faktoren in der Kardiologie [5] nennen die Autoren darüber hinaus speziell für die koronare Herzkrankheit (KHK) weitere psychosoziale Risikofaktoren:

- Schicht-, Nacht- und exzessive Mehrarbeit

- vitale Erschöpfung

- Angsterkrankungen, insbesondere phobische Angst und Panikstörungen

- posttraumatische Belastungsstörungen

- Schizophrenie

Zudem sind ausgeprägte akute Stresssituationen (z. B. Angst, Ärger, Trauer) häufige Auslöser von Myokardinfarkten.

Als psychosoziale Schutzfaktoren gegen Entstehung und ungünstige Verläufe einer KHK gelten gute soziale Unterstützung und das Zusammenleben in Ehe und Familie [5].

Demgegenüber sind bei der chronischen Herzinsuffizienz neben der Depression bzw. Depressivität weitere psychosoziale Risiko- oder Prognosefaktoren bislang nicht hinreichend gesichert. Allerdings findet sich eine akute Herzinsuffizienz im Rahmen von Tako-Tsubo- (oder Stress-) Kardiomyopathien (auch als „Broken-Heart“-Syndrom bezeichnet) deutlich gehäuft in akuten psychosozialen Belastungssituationen sowie bei Personen mit vorbekannten psychischen Störungen, insbesondere affektiven und Angststörungen [5,8].

Psychosoziale Faktoren wie Depressivität, Angst und TypD-Persönlichkeit (also die habituelle Neigung, unter negativen Affekten zu leiden bei gleichzeitiger sozialer Inhibition) erhöhen bei Herzpatienten das Risiko für ventrikuläre und/oder supraventrikuläre Herzrhythmusstörungen. Zudem kann ausgeprägter, akuter Stress ventrikuläre Tachykardien auslösen [5].

Unklar ist derzeit noch die Bedeutung psychosozialer Kausalfaktoren bei der Entstehung der arteriellen Hypertonie. Vielfach finden sich keine konsistenten linearen Zusammenhänge zwischen den untersuchten psychosozialen Faktoren und der Entstehung einer Hypertonie. Dies liegt vermutlich daran, dass der Blutdruck durch ein komplexes Feedbacksystem geregelt wird (siehe Kapitel „Psychophysiologische Effekte“).

\section{Grundlegende Mechanismen}

Für die beschriebenen psychosomatischen Zusammenhänge im Kontext von Herz-Kreislauf-Erkrankungen wurde mittlerweile eine Reihe von beteiligten Mechanismen identifiziert. Diese lassen sich grob einteilen in psychosoziale Effekte auf das Gesundheitsverhalten sowie „direkte“ psychophysiologische Effekte, die sich auf das Herz-Kreislauf-System auswirken. Beide Wege interagieren zudem miteinander und entwickeln sich entlang der individuellen Lebenslinie. 


\section{Gesundheitsverhalten}

Psychosoziale Risikofaktoren unterminieren die Motivation, sich an Behandlungsempfehlungen zu halten. Disstresserleben, Depressivität oder Ängste vor z. B. Medikamentennebenwirkungen oder Überforderung des Herzens durch Sport führen so selbst bei Einsicht in die Notwendigkeit eines empfohlenen Verhaltens zu Nonadhärenz gegenüber prognostisch günstigen Medikamenten und Verhaltensweisen. Einige Risikoverhaltensweisen, wie z. B. Frustessen oder Rauchen, dienen zudem kurzfristig der Emotionsregulation und können aus diesem Grund nicht ohne Weiteres aufgegeben werden [9]. Fehlernährung oder Rauchen haben ihrerseits physiologische Konsequenzen (z. B. Entwicklung eines metabolischen Syndroms, Vasokonstriktion), die mit psychophysiologischen Prozessen in der Krankheitsentstehung interagieren.

\section{Merke \\ Psychosoziale Risikofaktoren wirken als Barrieren gegen gesundheitsbewusstes Verhalten [2].}

\section{Psychophysiologische Effekte}

Stress und psychische Störungen beeinflussen die Funktion der neurohumoralen Stressachsen. Eine Störung der autonomen Balance bewirkt Funktionsänderungen an Herz und Gefäßen mit verstärkter kardialer Arbeit, Vasokonstriktion und Blutdruckerhöhung, was langfristig zu Gefäßschäden und einer Druckbelastung des Herzens führt. Die Herzfrequenzvariabilität nimmt ab, was die Anfälligkeit für Herzrhythmusstörungen erhöht. Zusätzlich führt die sympathische Aktivierung zu vermehrter Gerinnungsneigung und zur Aktivierung von Entzündungsprozessen, die u. a. in den Herzkranzgefäßen zur Entstehung von Plaques beitragen.

Bei vielen kardiovaskulär schädlichen psychophysiologischen Prozessen handelt es sich um Störungen selbstregulierter Feedbacksysteme. So führt etwa die stressbedingte Blutdruckerhöhung zunächst in aller Regel zu einer Barorezeptor-vermittelten Gegenregulation mit Aktivierung des Vagus und Absinken der Herzfrequenz, wodurch sich der Blutdruck wieder auf ein Normalmaß einpendelt. Allerdings hat die Barorezeptor-Aktivierung einen über die Blutdruckregulation hinausgehenden beruhigenden und schmerzlindernden Effekt auf das Zentralnervensystem, sodass die Blutdrucksteigerung als Mittel der Selbstberuhigung „gelernt“ werden und dadurch chronifizieren kann [10]. Auch beim Versagen der Gegenregulationsprozesse, etwa nach schwerer Traumatisierung bzw. bei posttraumatischer Belastungsstörung (z. B. [11]) oder bei chronischer Unterdrückung negativer Emotionen [12], scheint es zum gehäuften Auftreten einer Hypertonie zu kommen.

\section{Merke}

Gestörte Feedbackmechanismen sind häufig ursächlich an psychophysiologischen Prozessen beteiligt.
Wie die Blutdruckerhöhung kann auch die Entzündungsaktivierung auf das ZNS zurückwirken und das sogenannte „Sickness Behavior“ [13] auslösen, also einen depressionsähnlichen Zustand von Erschöpfung, Rückzug und Schmerzempfindlichkeit, der in einen Teufelskreis aus Stress und Inflammation münden kann.

\section{FALLBEISPIEL}

Teil 2

Zwei Jahre nach dem o. a. Ereignis wacht Herr M. nachts gegen 4 Uhr mit starken retrosternalen Schmerzen auf, die in Rücken und Unterkiefer ausstrahlen. Herr M. ist kaltschweißig und stark verängstigt. Er beruhigt sich zunächst etwas mit der Erklärung, dass er sich vermutlich nur den Magen verdorben habe und trinkt dagegen ein Glas Milch. Das hilft ihm wenig, was die Angst wieder größer werden lässt. Sollte es doch etwas mit dem Herzen sein? Ist es vielleicht wirklich so gefährlich, wie es sich anfühlt? Ob er einen Krankenwagen rufen soll? Aber es wäre doch peinlich, wenn am Ende nichts Ernstes vorliegt. Als Simulant möchte Herr M. nicht dastehen. Vielleicht wird es doch von selbst wieder besser. Bislang war ja auch nie etwas Schlimmes passiert.

Die Schmerzen setzen Herrn M. zu, aber er beißt die Zähne zusammen. Er läuft durch die Wohnung, versucht sich abzulenken. Dann steckt er sich eine Zigarette an, wie so oft in Stresssituationen. Aber die Schmerzen werden nicht besser. Er sollte wohl doch gleich morgens zum Hausarzt gehen. Als die Schmerzen auch um 7:30 Uhr noch nicht nachgelassen haben, macht er sich auf den Weg zum Hausarzt. Dort kollabiert er in der Wartezone. Das EKG zeigt einen akuten ST-Hebungs-Hinterwandinfarkt und einen intermittierenden AV-Block 3. Grades. Der Hausarzt veranlasst einen Notarzteinsatz mit Transport in die Klinik. Dort erfolgt umgehend eine Herzkatheteruntersuchung, in deren Rahmen ein großes verschlossenes Herzkranzgefäß wiedereröffnet und mit einem Stent versorgt wird. Herr M. wird nach der erfolgreichen Intervention auf die Überwachungsstation verlegt.

Das Auftreten von Herz-Kreislauf-Erkrankungen und die zugrunde liegenden physiologischen Störungen und Verhaltensweisen werden durch belastende Erfahrungen bzw. Traumatisierungen in Kindheit und Jugend mitbestimmt. Diese führen über z. T. epigenetische Prozesse zu einer Umprogrammierung des Organismus und hieraus erhöhter Stress- und Krankheitsanfälligkeit [14, 15]. Dies und die Erkenntnis, dass die Entwicklung kardiovaskulärer Erkrankungen häufig bereits im Jugend- oder jungen Erwachsenenalter einsetzt, unterstreichen die Bedeutung biografi- 
scher Faktoren in der Krankheitsentstehung, in der biologische, psychische und soziale Prozesse entlang der Lebenslinie miteinander interagieren [16].

\section{Somatopsychische Effekte kardiovaskulärer Erkrankungen auf das psychische Befinden}

\section{Grundlegende Mechanismen}

Psychologische Effekte von Herzkrankheiten und ihrer Behandlung

Durch eine Herzerkrankung werden die Patienten nahezu notwendigerweise mit Themen von Kontrollverlust und Todesdrohung konfrontiert. Hinzu kommen konkrete, oft beeinträchtigende Symptome wie Angina pectoris oder Luftnot und allgemein reduzierte Leistungsfähigkeit. Zusätzlich sehen sich Patienten vielfach mit der - für die Sekundärprävention wesentlichen, aber oft unpräzise formulierten - Forderung nach Veränderung des Lebensstils konfrontiert. Hinsichtlich deren Umsetzbarkeit besteht oft große Ungewissheit, z. B. wenn Patienten nicht wissen, wie intensiv sie sich bewegen dürfen oder gar sollen, ohne ihr Herz dabei zu überfordern. Meist ist eine regelmäßige Einnahme mehrerer Herzmedikamente notwendig, deren befürchtete oder real eintretende Nebenwirkungen die Lebensqualität beeinträchtigen können. Zudem erinnert die regelmäßige Medikamenteneinnahme täglich an die kardiale Vulnerabilität.

\footnotetext{
Merke

Die Rückwirkungen der Herzkrankheit auf das

subjektive Befinden können sowohl durch

psychologische als auch durch biologische

Mechanismen erklärt werden.
}

Notwendige, teilweise invasive Behandlungen mit begleitenden Ängsten und Schmerzen erfordern umfangreiche psychische Anpassungsleistungen. Dies gilt besonders für größere operative Eingriffe bis hin zur Herztransplantation sowie für die längerfristige Versorgung mit Kunstherzsystemen oder implantierten Defibrillatoren, insbesondere wenn Letztere wiederholt Stromstöße zur Beendigung von Herzrhythmusstörungen abgeben müssen.

Vielen Patienten gelingen diese Anpassungsprozesse, insbesondere bei gutem sozialen Rückhalt und ärztlicher Begleitung, mittelfristig gut. Bei einer relevanten Minderheit kommt es aber zu psychischen Störungen mit Krankheitswert, v. a. Anpassungs- und Belastungsstörungen, depressiven Episoden oder sekundären Herzangststörungen mit Somatisierungsneigung (siehe Kapitel „Psychische Komorbiditäten“). Umgekehrt findet sich nicht selten eine ausgeprägte Verleugnung und Delegation der Verantwortung für die Erkrankung an die behandelnden Ärzte oder gar die fatale Verkennung, dass nach der technischen „Reparatur“ einer Herzerkrankung wieder alles in Ordnung sei.

Physiologische Rückwirkungen auf das Befinden

\section{TIPP FÜR DIE PRAXIS}

Die morphologische Schwere einer Herzerkrankung korreliert allenfalls schwach mit der subjektiv wahrgenommenen Beeinträchtigung. Mehr als einzelne kardiale Messwerte spielen hierfür die Reaktion des gesamten Organismus auf die Herzerkrankung und bereits vorbestehende Regulationsstörungen die entscheidende Rolle.

Bei der Reaktion des gesamten Organismus spielen Entzündungsprozesse und ihre Wechselwirkung mit psychischen Faktoren (s.Kapitel „Grundlegende Mechanismen“) und eventuell auch Veränderungen im Darmmikrobiom eine wichtige Rolle.

Andererseits verfügt der Organismus über zahlreiche neurohumorale Gegenregulationsmechanismen, mit denen bei gestörter Herzfunktion die Homöostase im Organismus aufrechterhalten wird. Diese Mechanismen sind teilweise langfristig ungünstig und dienen daher als Ansatzpunkt medikamentöser Therapien mit z. B. Betablockern und ACE-Hemmern. Teilweise scheinen sie aber auch günstige Effekte zu haben, sodass ihre pharmakologische Verstärkung angestrebt wird. Hier ist insbesondere das kürzlich in die Herzinsuffizienztherapie eingeführte Sacubitril zu nennen, das den Abbau natriuretischer Peptide im Organismus hemmt. Aktuelle Daten sprechen dafür, dass die natriuretischen Peptide nicht nur günstige kardiale Effekte haben, sondern auch zur Stabilisierung des psychischen Befindens beitragen $[17,18]$.

\section{FALLBEISPIEL}

Teil 3

Nach dem Herzkatheter ist Herr M. erleichtert, dass die Schmerzen abgeklungen sind. Der behandelnde Arzt informiert ihn, dass er leider einen ausgedehnteren Herzinfarkt erlitten habe. Man habe das Schlimmste verhindern können, aber wahrscheinlich würde eine Störung der Pumpleistung des Herzens zurückbleiben. Außerdem bestünden noch weitere „mittelgradige“ Engstellen, die evtl. in Zukunft behandelt werden müssten.Wichtig sei, dass Herr M. in Zukunft regelmäßig mehrere Medikamente einnehme und seine Risikofaktoren in den Griff bekomme. Er müsse sofort mit dem 
Rauchen aufhören, sich gesund ernähren, regelmäßig Sport treiben und Stress vermeiden.

In dem 5-minütigen Gespräch ist weder für Nachfragen Zeit, noch besteht eine Gelegenheit, über die mit dem Infarktereignis verbundenen Gefühle zu sprechen, da der Arzt schon wieder in den Katheterraum muss. Anschließend kreisen die Gedanken in Herrn M.s Kopf. Was muss er jetzt alles machen? Welche Medikamente muss er jetzt nehmen? Warum sind die nötig? Haben sie eventuell schädliche Nebenwirkungen? Hoffentlich kann er sie schnell wieder absetzen. Und wie soll das mit der Ernährung, dem Sport und der Vermeidung von Stress alles funktionieren? Immerhin hat er verstanden, dass er jetzt wirklich mit dem Rauchen aufhören muss. Die Behandlung auf der Überwachungsstation ist dafür eine gute Gelegenheit und unter dem Schrecken des Infarkts beschließt Herr M., keine Zigarette mehr anzufassen. In den folgenden, jeweils sehr knappen Visiten findet Herr M. leider auch keine Gelegenheit, seine Verunsicherung und offenen Fragen anzusprechen. Er stimmt aber zu, im Anschluss an die Behandlung in eine Rehaklinik verlegt zu werden.

Im Abschlussgespräch wird ihm mitgeteilt, dass er Glück gehabt habe, den Infarkt überstanden zu haben. Leider betrage die Pumpleistung seines Herzens aber nur noch $40 \%$ und er müsse einige Medikamente lebenslang einnehmen. Das irritiert Herrn M., da er doch seit der Katheterintervention keine Beschwerden mehr hatte.

\section{Psychische Komorbiditäten und ihre Bedeutung für den Krankheitsverlauf}

Am besten gesichert ist der ungünstige prognostische Effekt einer Depression nach akutem Koronarsyndrom [3] sowie bei chronischer KH und Herzinsuffizienz. Vermutlich können depressive Symptome auch Rezidive von ventrikulären Tachykardien und Vorhofflimmern begünstigen.

Angststörungen und posttraumatische Belastungsstörungen sind nach akutem Infarkt ebenfalls mit beeinträchtigter Prognose assoziiert. Dagegen kann eine moderate Angstsymptomatik bei stabiler KHK evtl. prognostisch günstige Effekte zu haben, indem sie bspw. zu verbessertem Gesundheitsverhalten und vermehrter Inanspruchnahme ärztlicher Kontakte motiviert. Mutmaßlich gibt es bei Angststörungen sowohl ungünstige autonome als auch teilweise prognostisch günstige Verhaltenseffekte, sodass es von der Schwere und Aktivität der kardialen Erkrankung und der konkreten Angstsymptomatik abhängt, ob die Prognose positiv oder negativ beeinflusst wird.
Merke

Psychische Belastungen und Komorbiditäten wirken sich bei bestehender Herzerkrankung ungünstig auf Lebensqualität und somatischen Krankheitsverlauf aus.

Neben Morbidität und Mortalität stellt die gesundheitsbezogene Lebensqualität ein weiteres zentrales Therapieziel bei Patienten mit KHK und anderen Herzerkrankungen dar [4]. Sie hängt bei Herzpatienten deutlich stärker vom Ausmaß der psychosozialen Belastung bzw. dem Vorhandensein einer psychischen Komorbidität als von gängigen objektiven Maßen der kardialen Krankheitsschwere ab. Lediglich der klinische Schweregrad der Herzerkrankung, etwa die NYHA-Klasse der Herzinsuffizienz, hängt ähnlich eng mit der Lebensqualität zusammen wie Angst oder Depressivität. Zur Verbesserung der Lebensqualität müssen daher primär die klinischen Manifestationen der Herzerkrankung (Luftnot, Angina pectoris usw.) sowie das psychische Befinden gebessert werden, während von Maßnahmen, die lediglich auf kardiale Surrogatparameter oder Labor-Biomarker abzielen, allenfalls sehr geringe Effekte auf die Lebensqualität zu erwarten sind.

\section{FALLBEISPIEL}

\section{Teil 4}

In der Rehaklinik bemerkt Herr M., dass er noch deutlich geschwächt ist. Beim Ergometertraining bleibt seine Leistung hinter derjenigen einiger deutlich älterer Mitpatienten zurück, was ihn beschämt. „Da fühle ich mich doch wie ein alter Mann“. Wie soll er so im Beruf weiter seinen Mann stehen? Zum Glück hat er ja seinen Arbeitsplatz noch nicht verloren, aber wahrscheinlich nur, weil er sich immer als besonders zuverlässig und leistungswillig gezeigt hat. Wenn er jetzt wochenlang ausfällt und hinterher nicht mehr so kann wie bisher, könnte es doch noch Probleme mit dem Job geben.

Die medizinischen Informationsgespräche mit einem nur gebrochen Deutsch sprechenden Arzt kann Herr M. nur ansatzweise für sich nutzen. Vieles versteht er einfach noch nicht. Was bedeuten $40 \%$ Pumpleistung für seinen Alltag? Das scheint ziemlich wenig zu sein. Und wofür genau die einzelnen Medikamente nötig sind, kann er sich nicht merken. Zudem erfährt er von den Mitpatienten, dass die Medikamente Muskelschmerzen, Potenzprobleme und andere unangenehme Nebenwirkungen auslösen können. Ob die auch bei ihm auftreten werden? Wie kann er zukünftig wohl beim Sex noch seinen Mann stehen? Schon vor dem Infarkt war die Erektion schwächer geworden.Was sagt die Partnerin dazu, wenn es gar nicht mehr 
„klappt“? Sie hatte ihn im Krankenhaus besucht und ihm Mut gemacht - „das stehen wir gemeinsam durch“. Aber ob sie ihn als "Schlappschwanz“ noch haben will?

Zum Glück gibt es in der Rehaklinik 2 Psychologinnen. Ob man sich da melden darf? Und ob es nicht peinlich ist, als Mann im besten Alter über Ängste und Potenzprobleme mit einer jungen Frau zu reden? In den Patientenschulungen und im Stressbewältigungskurs machen die Psychologinnen einen netten Eindruck, aber bei so vielen Patienten haben sie sicher nicht für jeden Einzelnen Zeit.

Herr M. ist überrascht, aber auch etwas nervös, als er 3 Tage vor der Entlassung erfährt, dass die Psychologin Frau B. ihn gern am nächsten Tag zum Gespräch sehen möchte. Was soll er nur mit ihr besprechen? Er würde gern einige seiner offenen Fragen stellen, möchte aber nicht als Versager oder gar als verrückt dastehen.

Als die Psychologin das Gespräch mit den Worten „Wie geht es Ihnen?“ eröffnet, bricht Herr M. in Tränen aus. Das ist ihm seit der Zeit kurz nach Trennung von seiner Frau nicht mehr passiert. Wann hat ihn zuletzt jemand gefragt, wie es ihm geht? Und wie geht es ihm eigentlich? Irgendwie tut es gut, dass für die Tränen plötzlich ein Platz da ist. Der Psychologin ist sein Weinen offenbar nicht unangenehm. „Sie haben sicher in letzter Zeit einiges mitgemacht“, sagt sie.Herr M. nickt. „Es tut mir leid, dass ich mir bisher noch keine Zeit für Sie nehmen konnte. Mir war aufgefallen, dass Sie in den Gruppenterminen immer sehr zurückhaltend waren. Und der Fragebogen, den Sie bei der Aufnahme ausgefüllt hatten, zeigt, dass Sie offenbar seelisch sehr belastet sind“. Herr M. nickt wieder.

Im Gespräch gelingt es ihm dann, einige seine Ängste und Selbstzweifel anzusprechen. Auch das tut gut. Aber leider ist das Gespräch nach 20 Min vorbei. Als die Psychologin bedauert, dass sie leider bis zu seiner Entlassung keinen weiteren Termin frei habe, ist Herr M. enttäuscht. Es würde wohl guttun, mit ihr noch mehr Gespräche zu führen. So bleibt nur ihre Empfehlung, sich mit Unterstützung durch den Hausarzt um einen Platz für ambulante psychotherapeutische Gespräche zu bemühen. Aus der Reha wird Herr M. entlassen als vollschichtig arbeitsfähig für leichtere bis mittelschwere Tätigkeiten und mit der Empfehlung, an einem Reha-Nachsorgeprogramm teilzunehmen.

\section{Diagnostik psychosozialer Belastungen und Komorbiditäten bei Herzpatienten}

\section{ALLGEMEINE VORGEHENSWEISE}

Grundsätzlich wird die Diagnose einer psychischen Komorbidität bei Herzpatienten ebenso gestellt wie bei jedem anderen Patienten, d.h. in der Regel auf der Basis eines klinischen Interviews. Hierbei ist aber auf die Überlappung von möglichen Symptomen psychischer und kardialer Erkrankungen zu achten, die gegebenenfalls zu Fehleinschätzungen führen kann.

Auf Überlappungen psychischer und kardialer Symptome ist zu achten: So werden gelegentlich supraventrikuläre Tachykardien mit begleitender Angstsymptomatik irrtümlich als primäre Panikstörung gedeutet, wie auch Sinustachykardien im Rahmen von Panikattacken gelegentlich mit Herzrhythmusstörungen verwechselt werden. Eine genaue Exploration ist auch bei Brustschmerzen, allgemeiner Leistungsminderung, Schlaf- oder Appetitstörungen erforderlich, die Symptome sowohl der kardialen als auch einer psychischen oder funktionellen Erkrankung sein können. Zudem kann z. B. psychischer Stress sowohl zu unspezifischen Thoraxschmerzen als auch zur Auslösung „echter“ Angina pectoris führen.

\section{TIPP FÜR DIE PRAXIS}

Oft übersehen wird die Diagnose F54 nach ICD-10 („psychische und Verhaltensfaktoren bei andernorts klassifizierten Erkrankungen“). Sie ist in den häufigen Fällen zu codieren, in denen Entstehung und Verlauf einer Herzerkrankung sowie resultierende Beschwerden oder Entgleisungen von Risikofaktoren wie Hypertonie oder Diabetes offensichtlich auf psychische und Verhaltensfaktoren zurückgeführt geführt werden können.

Wichtig ist es, auch auf krankheitsreaktive psychische Störungen wie Anpassungsstörungen, sekundäre Somatisierung und posttraumatische Symptome bis hin zu posttraumatischen Belastungsstörungen zu achten.

Neben der Störungsdiagnostik wird die Exploration des subjektiven Krankheitserlebens und ein routinemäßiges Screening auf psychosoziale Risikofaktoren schon im kardiologischen Setting empfohlen [5]. Hierfür eignen sich Screeningfragen in der Anamnese und validierte Selbstbe- 
urteilungsfragebögen für z. B. Depressivität oder Angst, zu denen in den Leitlinien konkrete Empfehlungen gegeben werden $[2,4]$.

Umgekehrt sollte auch im Rahmen einer psychotherapeutischen Eingangsdiagnostik die reale Ebene der kardialen Erkrankung sowie das Vorhandensein von Risikofaktoren, die psychoökonomische Funktion von Risikoverhaltensweisen und die Bereitschaft zur Lebensstiländerung exploriert und bei der Generierung von psychodynamischen Hypothesen oder Verhaltensanalysen angemessen berücksichtigt werden.

\section{Merke \\ Bei der Diagnostik sind sowohl die krankheitsreaktiven Auswirkungen auf die Psyche, als auch die psychischen Einflussfaktoren auf die somatische Ebene zu berücksichtigen.}

Zum Nachweis eines Zusammenhangs zwischen psychischen Belastungen und Herz-Kreislauf-Regulationsstörungen ist es z. B. hilfreich, Patienten während der Registrierung einer 24-Stunden-EKG- oder Blutdruckmessung ein genaues Protokoll führen zu lassen, in dem neben körperlichen Aktivitäten auch interpersonelle Ereignisse und emotionale Auslenkungen notiert werden. Unter Laborbedingungen sind darüber hinaus psychophysiologische Untersuchungen mit standardisierten Stresstests oder Entspannungsübungen und gleichzeitiger Registrierung unterschiedlicher Herz-Kreislauf-Parameter verfügbar.

\section{FALLBEISPIEL}

\section{Teil 5}

Der Hausarzt ist nach der dramatischen Infarktsituation erleichtert, Herrn M. am Tag nach der Entlassung aus der Reha in äußerlich guter Verfassung wiederzusehen. Allerdings ist das Wartezimmer sehr voll und neben der Rezeptausstellung bleibt nur wenig Zeit für ein Gespräch. Insbesondere beschäftigt Herrn M., wie er denn in der kommenden Woche wieder arbeiten gehen soll. Das kann er sich noch überhaupt nicht vorstellen. Er fühlt sich noch immer geschwächt und befürchtet, durch den Stress bei der Arbeit und die anstrengende Tätigkeit erneut einen Herzinfarkt zu bekommen. Der Hausarzt stellt ihm daher eine Arbeitsunfähigkeitsbescheinigung für 2 Wochen aus und vereinbart einen Termin zur weiteren Besprechung für die kommende Woche.

Im nächsten Termin geht es zunächst noch einmal darum, warum Herr M. welche Medikamente nehmen muss. So kann Herr M. ihren Nutzen etwas besser verstehen, aber er äußert auch Sorgen vor den Nebenwirkungen, auf die der Hausarzt ausgiebig eingeht. Da bislang keine wesentlichen Nebenwirkungen aufgetreten sind, stimmt Herr M. zu, sie vorerst weiter zu nehmen und sich bei eventuellen Nebenwirkungen zu melden. Dann geht es um das Gesundheitsverhalten. Herr M. ist stolz darauf, dass er bislang noch nicht wieder mit dem Rauchen angefangen hat, sorgt sich aber, ob es mit dem Nichtrauchen auch klappt, wenn er wieder täglich den Stress bei der Arbeit ertragen muss. Noch schwerer fällt es ihm, sich vorzustellen, wie er seine Ernährung umstellen kann. Zeit, sich etwas Gesundes zu kochen, hat er neben der Arbeit nicht - und er hat es auch nie gelernt. Was kann er sich beim Sport zutrauen? Leider wird vor Ort keine intensivierte Rehanachsorge angeboten. In der Reha durfte er sich zuletzt mit $100 \mathrm{~W}$ belasten aber was bedeutet das im Alltag? Und ist es nicht gefährlich, wenn kein Arzt in der Nähe ist? Da waren doch noch mehr Engstellen an den Kranzgefäßen.

Das Gespräch über die Psychotherapie findet hinter all diesen drängenden Themen keinen Platz. Dazu kommt es erst 3 Wochen später, als Herr M. die AU-Bescheinigung zum 2.Mal verlängert bekommen möchte und der Hausarzt nachfragt, warum Herr M. sich denn immer noch nicht zutraue, zur Arbeit zu gehen. Die Krankenkasse hat sich bei ihm gemeldet, dass sie aufgrund des Rehaberichts eine weitere Krankschreibung nicht akzeptiere. Auf genaues Nachfragen berichtet Herr M. dem Hausarzt, er habe gar keine Energie, schlafe nachts schlecht, grübele und komme morgens schlecht aus dem Bett. Die Stimmung sei gedrückt, er könne sich zu vielem, etwa zu körperlichen Aktivitäten, nicht aufraffen, hänge die Tage über vor dem Fernseher oder PC, gehe kaum aus dem Haus und habe sich auch noch nicht wieder getraut, mit seiner Partnerin zu schlafen - aus Angst zu „versagen“ oder seinem Herzen zu schaden. Das Herz stolpere manchmal und das erschrecke ihn jedes Mal. Die Psychologin in der Reha habe zu einer Psychotherapie geraten. Der Hausarzt begrüßt diesen Vorschlag und nennt Herrn M. einige Namen von Therapeuten, bei denen er sich wegen eines Therapieplatzes melden solle. Zur Überbrückung verordnet er Sertralin, da Herr M. vermutlich eine Depression habe.

\section{Behandlung und Prävention}

\section{Psychosomatische Ansätze}

Die meisten Herzpatienten werden zunächst von Hausärzten, Kardiologen oder Rehamedizinern behandelt. In diesem Rahmen spielt sowohl die psychosomatische Grundversorgung als auch eine Mitbehandlung durch ärztliche 
oder psychologische Psychotherapeuten im Rahmen von Konsiliar-und Liaisonmodellen eine große Rolle [5].

\section{Merke}

Wichtig ist bei jedem Fall eine möglichst widerspruchsfreie abgestimmte Kommunikation der unterschiedlichen Behandler mit dem Patienten.

Sofern keine regelmäßige Präsenz eines psychotherapeutischen Teammitglieds gewährleistet werden kann, ist entscheidend, dass die somatisch behandelnden Ärzte eine psychosoziale Problematik oder psychische Komorbidität orientierend erfassen, im Verlauf im Blick behalten und gegebenenfalls eine zusätzliche psychosomatischpsychotherapeutische Mitbehandlung veranlassen.

Mit patientenzentrierter Kommunikation und psychosomatischer Grundversorgung können viele Patienten mit unspezifischen psychosozialen Risikofaktoren oder leichteren psychischen Störungen auf der Basis einer tragfähigen Arzt-Patient-Beziehung auch innerhalb der allgemeinmedizinischen oder kardiologischen Routine erfolgreich behandelt werden.

Merke

Beziehungskonstanz mit den Behandlern ist wesentlich - sowohl zur oft schwierigen Umsetzung von Verhaltensänderungen im Alltag als auch bei der Bewältigung chronischer Herzerkrankungen.

Für die konstante Arzt-Patient-Beziehung sind quartalsweise Arztkontakte mit begrenzter Gesprächsdauer oft ungenügend. Bei Bestehen einer psychischen Komorbidität oder einer ausgeprägten psychischen und Verhaltenskomponente der Herzkrankheit ist daher häufig eine zusätzliche ambulante Psychotherapie indiziert.

Psychosoziale Interventionen einschließlich spezifischer psychotherapeutischer Behandlungen erreichen allerdings in Studien an Herzpatienten deutlich geringere Effektstärken als in Psychotherapiestudien an Herzgesunden. Ob sie in der Lage sind, auch den Verlauf der Herzerkrankung günstig zu beeinflussen, ist ungeklärt.

Im Rahmen der kardiologischen Rehabilitation werden an psychologischen Interventionen u. a. Stressbewältigungstrainings, Patientenschulungen zur Risikofaktorkontrolle und psychologische Einzelgespräche angeboten. Ältere Metaanalysen legen nahe, dass diese Interventionen sich nicht nur auf das Befinden, sondern möglicherweise auch auf den Krankheitsverlauf günstig auswirken. Allerdings sind die eingesetzten Interventionen sehr heterogen. Inwieweit sie auch neben unspezifischen Rehaeffekten und körperlichem Training zusätzliche Effekte haben, wird gegenwärtig untersucht.

\section{Aktuelle Studienlage}

Spezifische Psychotherapien

Hinsichtlich spezifischer Psychotherapien in der Depressionsbehandlung bei KHK-Patienten erbrachte in der CREATEStudie eine 3-monatige interpersonelle Psychotherapie im Vergleich zu einer unspezifischen wöchentlichen Gesprächsintervention (vergleichbar einer psychosomatischen Grundversorgung) keinen Vorteil [19]. Eine kognitive Verhaltenstherapie gegen Depression oder niedrige soziale Unterstützung zeigte in der ENRICHD-Studie nur einen sehr kleinen Therapieeffekt auf depressive Symptome [20]. Auch für eine gestufte kombiniert psychodynamisch-kognitiv-verhaltenstherapeutische Intervention konnte an depressiven KHK-Patienten in der SPIRR-CADStudie kein eindeutiger Effekt und nur in der Untergruppe mit Typ-D-Persönlichkeit ein positiver Trend gefunden werden [21]. Kleine positive Effekte bei depressiven Patienten mit Herzinsuffizienz werden in einer aktuellen Metaanalyse [22] für kognitive Verhaltenstherapie beschrieben, die auch in der ESC-Herzinsuffizienz-Leitlinie [6] empfohlen wird. Die aktuelle deutsche Depressionsleitlinie empfiehlt auch für depressive KHK-Patienten eine Psychotherapie [23]. Die Datenbasis ist aber bis heute unbefriedigend.

\section{Antidepressive Pharmakotherapie}

Auch für antidepressive medikamentöse Behandlungen bei Herzpatienten sind die Befunde uneindeutig: Während es für Koronarpatienten mit wenigstens mittelgradiger, insbesondere rezidivierender Depression Hinweise auf eine antidepressive Wirksamkeit von Sertralin (SADHART; [24]), Citalopram (CREATE; [19]) und Escitalopram (EsDEPACS; [25]) gibt, zeigten sich sowohl Sertralin (SADHART-CHF; [26]) als auch Escitalopram (MOOD-HF; [27]) bei Patienten mit Herzinsuffizienz einer Placebomedikation weder hinsichtlich des Depressionsverlaufs noch hinsichtlich der medizinischen Krankheitsendpunkte überlegen. Eine aktuelle Langzeit-Follow-up-Untersuchung der monozentrischen koreanischen EsDEPACS-Studie [28] zeigt als erster RCT an Koronarpatienten einen möglichen prognostischen Nutzen einer antidepressiven Medikation mit Escitalopram und keine ungünstigen kardialen Nebenwirkungen.

Cave
Höhere Dosierungen von Citalopram sollten bei
Herzpatienten aufgrund der Gefahr von QTc-Verlänge-
rungen im EKG und damit verbundenem erhöhten
Arrhythmierisiko allenfalls unter sorgfältiger Kontrolle
eingesetzt werden.




\section{FALLBEISPIEL}

Teil 6

Die Therapieplatzsuche gestaltet sich schwierig. Oft spricht Herr M. vergeblich auf Anrufbeantworter, was ihn zunehmend entmutigt. Nach mehreren Wochen bekommt er einen Erstgesprächstermin bei einem psychologischen Psychotherapeuten. Der Therapeut ist freundlich und bestätigt Herrn M., dass er an einer Depression leide. Er vermittelt ihm Verständnis für seine schwierige Lage, wirkt aber bezüglich der ernsten Herzerkrankung unsicher und kann einige Fragen zu medizinischen Themen nicht beantworten. Das irritiert Herrn M. und so nimmt er den vereinbarten 2. Termin nicht wahr. Auch das Sertralin hat er zwischenzeitlich wegen MagenDarm-Beschwerden wieder abgesetzt.

Auf das Drängen des Hausarztes hatte er seine Arbeit 8 Wochen nach dem Infarkt wieder aufgenommen. Er macht sich erneut auf die Suche nach einem Psychotherapieplatz. Im Erstgespräch mit einem psychosomatischen Facharzt fühlt er sich auch mit seiner Herzkrankheit gut aufgehoben. Leider stellt ihm der Therapeut eine reguläre Therapie erst für in 5 Monaten in Aussicht. Einige Wochen später erleidet er auf dem Weg zur Arbeit eine Attacke mit Herzklopfen, Schmerzen in der Herzgegend, Schweißausbruch und Kurzatmigkeit sowie starkem Angstgefühl, weshalb er diesmal umgehend die Notaufnahme aufsucht. Dort wird ein erneuter Herzinfarkt ausgeschlossen und Herr M. ohne Befund und mit der Empfehlung entlassen, sich ambulant beim Kardiologen vorzustellen. Ein Termin dort nach einigen Wochen zeigt weiterhin eine mäßig eingeschränkte Pumpfunktion, aber keinen klaren Hinweis auf Durchblutungsstörungen. Der Kardiologe rät Herrn M. dazu, die von der Klinik verordnete Medikation fortzuführen, bei noch nicht optimal eingestelltem Blutdruck lediglich die Dosierung anzupassen und sich einer ambulanten Herzsportgruppe anzuschließen. Eine erneute Katheteruntersuchung hält er nicht für erforderlich. Als Herr M. seine Ängste wegen der verbliebenen Engstellen an den Kranzgefäßen anspricht, erfährt er nur, das sei im Grunde kein Problem, aber völlige Sicherheit gebe es natürlich nie. In den folgenden Wochen treten noch mehrmals Anfälle mit „Herzbeschwerden“ auf, wobei sich jeweils kein Infarkt nachweisen lässt. Herr M. ist mittlerweile schon wieder seit mehreren Wochen arbeitsunfähig. Bei der 4 . Vorstellung auf der Notaufnahme wird dann „sicherheitshalber“ doch noch einmal ein Herzkatheter durchgeführt und ein Stent in eine der verbliebenen Engstellen eingebracht.
Dennoch kommt es weiterhin zu ähnlichen, teilweise weniger stark ausgeprägten Attacken mit herzbezogenen Beschwerden (insbesondere in öffentlichen Situationen) und zunehmendem Vermeidungsverhalten. Beim 5. Besuch auf der Notaufnahme wird der psychosomatische Konsiliararzt hinzugezogen, der die Übernahme auf die internistisch-psychosomatische Station veranlasst. Der Infarkt ist mittlerweile 8 Monate her.

Unter selektiven Serotonin-Wiederaufnahmehemmern muss bei Patienten mit Einnahme von Thrombozyten-Aggregationshemmern oder Antikoagulanzien generell mit einem erhöhten, vorwiegend gastrointestinalen Blutungsrisiko sowie mit Adhärenzproblemen aufgrund unerwünschter Nebenwirkungen wie Magen-Darm-Beschwerden, sexuellen Funktionsstörungen usw. gerechnet werden.

Vom Einsatz trizyklischer Antidepressiva bei Herzpatienten wird wegen des ungünstigen Nebenwirkungsprofils in aller Regel abgeraten [4, 5].

\section{Psychokardiale Behandlungsverfahren}

Für Patienten mit ausgeprägter Interaktion psychosozialer Risikofaktoren mit der kardialen Erkrankung oder gravierenden psychischen oder sozialen Folgeproblemen haben sich in einigen Akut- und Rehabilitationskliniken multimodale psychokardiologische Behandlungsangebote entwickelt. Dort können Patienten zugleich eine kardiologische Behandlung, Psychotherapie, Sozialberatung und weitere komplementäre Behandlungsverfahren angeboten werden - Angebote, die deutlich über das Ausmaß an psychologischer Unterstützung im Rahmen konventioneller kardiologischer Rehabilitation hinausgehen und eine deutlich höhere und vielfältigere Therapiedosis pro Zeiteinheit anbieten können als eine Richtlinien-Psychotherapie. Erste unkontrollierte Studien deuten auf gute Wirksamkeit dieser Angebote hin. Eigene Erfahrungen zeigen, dass insbesondere das abgestimmte Miteinander kardiologischer und psychotherapeutischer Behandlung sowie körpertherapeutische Elemente, die den Patienten wieder ein Vertrauen in den kranken Körper vermitteln können, von den Patienten als sehr hilfreich erlebt werden [29]. Auch eine antidepressive Medikation kann ggf. unter engmaschiger Überwachung eindosiert werden.

In der Phase der chronischen KHK ist eine längerfristige und regelmäßige Begleitung im Umgang mit verhaltensabhängigen Risikofaktoren - und ggf. eine ambulante Psychotherapie - erforderlich. Hier ist es wichtig, in guter sein Statin aus Angst vor Nebenwirkungen „nur gelegentlich“ einzunehmen. In der 24-Stunden-Blutdruckmessung zeigen sich insbesondere in emotional belastenden Situationen noch erhöhte Werte bis 170/95 mmHg. Die 24-Stunden-Mittelwerte sind dagegen mit 138/85 mmHg nur leicht erhöht, 
die Nachtabsenkung regelrecht mit einigen erhöhten Werten während Wachphasen mit intensivem Grübeln.

\section{FALLBEISPIEL}

\section{Teil 7}

Auf der Station wirkt Herr M. antriebsgemindert, depressiv herabgestimmt und zeigt ein deutliches Sicherheits- und Vermeidungsverhalten. Im Kontakt schwankt er zwischen Klagsamkeit und übertriebener Selbstdarstellung in der Patientengruppe mit angepasstem Verhalten gegenüber dem Einzeltherapeuten und latent feindseliger, teilweise abwertender Haltung gegenüber dem Pflegeteam und der Kunsttherapeutin.

Es wird eine mutmaßlich rezidivierende depressive Störung, gegenwärtig mittelgradige Episode (ICD-10 F33.1) sowie eine sekundäre Herzangststörung (hier nach ICD-10 eine Agoraphobie mit Panikstörung F40.01) sowie psychische und Verhaltensfaktoren (F54) bei arterieller Hypertonie und koronarer Herzkrankheit diagnostiziert.

In der biografischen Anamnese fällt der frühe Tod der als weich und liebevoll geschilderten Mutter an einer Brustkrebserkrankung im 11. Lebensjahr des Patienten und ein Herzinfarkt des als leistungsorientiert und teilweise cholerisch geschilderten Vaters im Alter von 50 Jahren auf. Der Vater habe sich aber nicht unterkriegen lassen und weiter gearbeitet, bis er mit Ende 50 an einem 2. Infarkt verstarb. Nach dem Tod des Vaters habe es mit dem 3 Jahre jüngeren Bruder eine heftige Auseinandersetzung um das Erbe gegeben, woraufhin Herr M. den Kontakt abbrach. Nach Hauptschulabschluss hatte Herr M. eine Bäckerlehre absolviert, den Beruf jedoch bald wegen einer Allergie nicht mehr ausüben können. Daher habe er mit 25 Jahren in der Fabrik angefangen, in der er noch immer beschäftigt sei, viele Jahre davon auch im Schichtdienst sowie seit Jahren in ständiger Sorge um den Arbeitsplatz und mit starker Konkurrenz unter den Kollegen.

Mit 26 Jahren lernte er seine erste Frau, eine attraktive Friseurin, kennen. Er selbst sei damals auch sportlich und auf gutes Aussehen bedacht gewesen. Mit der Frau bekam er 2 Kinder. Er habe gar nicht bemerkt, dass es in der Ehe Probleme gegeben habe und sei von der Trennung der Frau vor $6 \frac{1}{2}$ Jahren völlig überrascht gewesen. Er habe eine ganze Weile gebraucht, um über die Trennung hinwegzukommen. Mittlerweile sei nach langen Auseinandersetzungen die Scheidung erfolgt. Kurz vor dem Infarkt habe noch einmal eine Gerichtsverhandlung stattgefunden, die ihn sehr aufgewühlt habe. Jetzt sei alles einigermaßen geregelt und den 18-jährigen Sohn begleite er gelegentlich zum Fußball, aber er leide nach wie vor daran, mit der 15-jährigen Tochter nur wenig Kontakt zu haben. Weiterhin unterhalten er und die neue Partnerin getrennte Wohnungen, auch wenn sie nach dem Infarkt überlegt haben, evtl. zusammenzuziehen, zumal die 16-jährige Tochter der Partnerin demnächst zu einer Ausbildung in ein auswärtiges Wohnheim ziehen wird. Die Beziehung hat sich seit dem Infarkt vertieft, aber Herr M. sorgt sich, der Partnerin zur Last zu fallen. Zudem habe er sich seit dem Infarkt noch immer nicht getraut, wieder mit der Partnerin sexuell intim zu werden.

Somatisch findet sich im Labor ein noch zu hoher LDL-Cholesterin-Spiegel. Auf die Medikation angesprochen räumt Herr M. ein,

Das angebotene Antidepressivum lehnt Herr M. zunächst aufgrund seiner negativen Vorerfahrungen pauschal ab. Im Verlauf stimmt er einer niedrig dosierten MirtazapinMedikation zur Verbesserung des Nachtschlafs zu und kann hiervon subjektiv profitieren. Die indizierten Laborund EKG-Kontrollen tragen zu seiner Beruhigung bezüglich des Medikaments bei.

Unter Einzel- und Gruppenpsychotherapie, Körper- und Trainingstherapie, Kunsttherapie, psychoedukativen Gruppen sowie regelmäßigen Entspannungsübungen und Anpassung der Blutdruckmedikation gelingt es Herrn M. allmählich, sich auf das therapeutische Angebot einzulassen. Er unternimmt zunächst in Begleitung, dann zunehmend allein Spaziergänge, geht wieder einkaufen und fühlt sich unter der Trainingstherapie allmählich wieder etwas leistungsfähiger. Der Schlaf bessert sich ebenso wie Stimmung und Antrieb. In einem Paargespräch wird das Thema der bisher vermiedenen Sexualität und die Frage einer eventuellen gemeinsamen Wohnung thematisiert. Dabei wird deutlich, dass beide Partner sehr vorsichtig miteinander umgehen. Sie werden ermutigt, Pläne für gemeinsame, z. B. sportliche, Aktivitäten zu entwickeln.

In einem Gespräch mit der Sozialarbeiterin werden erste Lösungsideen für die berufliche Situation entwickelt. Herr M. will nach Entlassung eine Wiedereingliederung nutzen, ein Gespräch mit seinem Vorgesetzten führen und ihn um Versetzung in einen körperlich weniger belastenden Bereich der Firma bitten, nachdem ihm bedeutet wurde, dass aktuell wegen der guten Auftragslage keine Entlassungen geplant würden.

Panikattacken treten auf der Station und auch während der Expositionsübungen nicht auf, leichtere Angstzustände kann er mit Unterstützung des Pflegeteams zunehmend besser bewältigen. 
Bei Entlassung hat sich die psychische Symptomatik deutlich gebessert. Herr M. hat nach mehreren ausführlichen ärztlichen Gesprächen über die Herzkrankheit besser verstanden, dass ein Großteil der Verantwortung für den weiteren Krankheitsverlauf bei ihm liegt und er kann diese Verantwortung immer besser annehmen. Er hat sich nach den positiven Erfahrungen in der Körper- und Trainingstherapie für eine Herzsportgruppe angemeldet und den niedergelassenen Psychosomatiker um einen möglichst frühen Termin für den Therapiebeginn gebeten. Seine Medikamente nimmt er nun regelmäßig, was sich bereits im LDL-Cholesterin-Spiegel zeigt. Zudem ist es ihm weiterhin gelungen, nicht zu rauchen.

Trotz dieses guten therapeutischen Ergebnisses bleibt offen, ob es ihm gelingt, seine Vorsätze im Alltag langfristig umzusetzen.

Abstimmung zwischen Hausarzt, Kardiologen und ggf. Psychotherapeut die Patienten in ihrer Eigenverantwortung zu stärken und wo nötig zu unterstützen.

Im langfristigen Verlauf kann eine abgestimmte Behandlung dazu beitragen, den oft unvermeidlichen Progress der Erkrankung mit hohem Risiko für die Zunahme der Veränderungen an den Herzkranzgefäßen, Ausbildung einer ausgeprägteren Herzinsuffizienz oder Auftreten behandlungsbedürftiger Herzrhythmusstörungen frühzeitig zu erkennen und mit dem Patienten adäquat darauf zu reagieren. Auch wenn im Einzelfall ein prognostischer Nutzen dieser Maßnahmen nicht eindeutig feststeht, kann eine koordinierte Behandlung maßgeblich zum Erhalt der Lebensqualität beitragen.

\section{Merke \\ Die empfehlenswerte engmaschige Begleitung zwischen Hausarzt, Kardiologen und ggf. Psychotherapeut scheitert leider oft an Kapazitätsproblemen im Gesundheitssystem.}

Relativ gute Belege gibt es für das in den USA ursprünglich für Patienten mit psychischen Störungen entwickelte und teilweise bereits etablierte alternative Modell der „Collaborative Care“ [30]. Hierbei werden Pflegekräfte als Behandlungsassistentinnen eingesetzt, die einen regelmäBigen Kontakt mit den Patienten pflegen und mit ihnen Behandlungsziele und konkrete Schritte vereinbaren wie die Aktivierung von Selbsthilfepotenzialen, kurze psychotherapeutische Interventionen oder die Anpassung und regelmäßige Einnahme antidepressiver Medikamente. Die Behandlungsassistentin steht unter regelmäßiger Supervision durch ein medizinisch-psychotherapeutisches Expertenteam, dessen Empfehlungen sie den Patienten und ihren behandelnden Ärzten übermittelt.

Mehrere US-Studien konnten zeigen, dass damit auch bei Herzpatienten eine relevante Besserung depressiver Sym- ptome zu erreichen ist, wobei vermutlich mehr die persönliche Betreuung als die Veränderung der antidepressiven Medikation eine Rolle spielt [31]. Eine der USStudien [32] erweiterte das Modell der Collaborative Care zu einer gleichzeitigen Behandlung sowohl der Depression als auch des kardiovaskulären Risikoprofils und konnte zeigen, dass auch einige verhaltensabhängige Risikofaktoren durch eine solche „Blended Collaborative Care“ bzw. „TeamCare“-Intervention günstig zu beeinflussen waren. In einer großen Praxis-Implementierungsstudie (COMPASS; [33]) ließen sich diese Ergebnisse unter Routinebedingungen weitgehend reproduzieren. Aus Deutschland legt eine erste Pilotstudie nahe, dass sich ein solches therapeutisches Konzept auch hierzulande erfolgreich umsetzen lässt [34]. In der Praxis ist es allerdings bislang leider nicht verfügbar.

\author{
Merke \\ Das in den USA etablierte Modell der Blended \\ Collaborative Care scheint auch in Deutschland \\ umsetzbar zu sein.
}

\section{Wissenschaftliche Evidenz und klinische Praxis - zwei Welten?}

Das geschilderte Fallbeispiel zeigt einen Ausschnitt aus einem typischen Krankheits- und Behandlungsverlauf eines Koronarpatienten.

Herr M. weist mit niedrigem sozioökonomischem Status, Stress im Beruf und Familienleben, Schichtarbeit sowie mangelhafter sozialer Unterstützung und einer mutmaßlichen Depressionsanamnese mehrere gesicherte psychosoziale Risikofaktoren aus, die zudem einhergehen mit gesundheitsschädlichen Verhaltensweisen (Rauchen zur Stressregulation, Fehlernährung, Bewegungsmangel) und physiologischen Folgen (Übergewicht, durch Stress mitbedingte und angesichts mangelnder Adhärenz insuffizient eingestellte Hypertonie). Die Mischung aus Angst und Verleugnung führte zu mangelhafter Inanspruchnahme ärztlicher Behandlungen im Vorfeld des Infarkts wie auch zu verzögertem Hilfesuchverhalten mit beinahe tödlichen Folgen in der Infarktsituation. Aufgrund der Verzögerung konnte auch ein teilweiser Funktionsverlust des Herzmuskels nicht mehr verhindert werden. Möglicherweise hätte ein frühzeitigeres proaktives Vorgehen mit Etablierung eines stabilen Arzt-Patient-Verhältnisses und Begleitung bei den indizierten Untersuchungen und Verhaltensänderungen bereits im Vorfeld präventiv wirksam werden können. Retrospektiv lässt sich der Infarkt in Übereinstimmung mit der Literatur zeitlich mit der emotional belastenden Scheidungsverhandlung in Verbindung bringen.

In der Behandlung nach dem Infarkt offenbaren sich viele typische Bruchstellen, an denen Best-Practice-Empfehlungen der Leitlinien und Positionspapiere aus verschiedenen 
Gründen nicht umgesetzt wurden: In der kardiologischen Akutklinik fand nach adäquater Akutversorgung unter dem herrschenden Zeitdruck nur eine sehr oberflächliche und wenig patientenzentrierte Aufklärung statt. Emotionale Themen und Fragen der Alltagskonsequenz wurden implizit an die Rehaklinik delegiert. Immerhin konnte Herr M. durch den klaren ärztlichen Ratschlag zur Rauchabstinenz motiviert werden.

Merke

Zeitmangel und eine insgesamt wenig patientenzentrierte Aufklärung über Sorgen und Ängste der Patienten bestimmen häufig den Behandlungsalltag und verzögern eine adäquate psychosomatisch orientierte Behandlung.

Trotz positiven Fragebogenscreenings erfolgte in der Reha erst kurz vor Entlassung ein einmaliges kurzes psychologisches Einzelgespräch, sodass die „narzisstische Krise“ (vgl. [35]) mit Verlust der körperlichen Integrität, Schwächeerleben und zunehmendem Zusammenbruch der Verleugnung nicht mehr ausführlich thematisiert werden konnte. Bei einem Schlüssel von laut Vorgaben der DRV nur 1,25 Psychologenstellen auf 100 kardiologische Rehabilitanden erhält die Mehrzahl der Patienten in der 3-wöchigen Reha gar kein Einzelgespräch. Zum Entlassungszeitpunkt lag bei Herrn M. vermutlich mindestens eine ängstlich-depressive Anpassungsstörung vor. Hierzu kann evtl. auf biologischer Ebene auch die inflammatorische Aktivierung der Postinfarktphase und auf psychologischer Ebene neben den vorbestehenden Belastungen die Erinnerung an die Herzkrankheit und den frühen Herztod des Vaters mit beigetragen haben.

\section{KERNAUSSAGEN}

- Psychische, soziale und Verhaltensfaktoren tragen zur Entstehung von Herz-Kreislauf-Krankheiten bei und sollten möglichst frühzeitig identifiziert und gemeinsam mit den Betroffenen angegangen werden.

- An der Auslösung akuter kardialer Ereignisse und der oft verspäteten Inanspruchnahme ärztlicher Hilfe sind psychische und interpersonelle Faktoren ebenfalls häufig beteiligt.

- Die Herzkrankheit selbst stellt über psychosoziale und biologische Mechanismen einen erheblichen Stressor dar und führt häufig zu psychischen Folgestörungen, die ihrerseits Lebensqualität und Prognose verschlechtern.

- Wichtig ist in allen Phasen der Behandlung eine klare, widerspruchsfreie, empathische und kontinuierliche Kommunikation mit dem Patienten und zwischen den Behandlern sowie die rechtzeitige Erkennung und Behandlung psychischer Störungen oder maladaptiver Verhaltensweisen (z. B. Nonadhärenz).

- Sowohl für psychotherapeutische Behandlungen als auch für eine antidepressive Medikation liegen bei Herzpatienten mit psychischer Komorbidität im Vergleich zu anderen psychisch kranken Patientengruppen erst wenige und uneindeutige Studienergebnisse vor.

- Bei unzureichender Wirksamkeit einer psychosomatischen Grundversorgung - oft sinnvollerweise ergänzt durch körperliches Training - basiert die Indikationsstellung spezifischer Behandlungen auf den Patientenpräferenzen, der Verfügbarkeit konkreter psychotherapeutischer Angebote und der Nutzen-Risiko- Abwägung antidepressiver Medikamente.

- In schweren Fällen kann eine spezialisierte internistisch-psychosomatische oder psychokardiologische Reha- oder Krankenhausbehandlung erforderlich werden. Hieran sollten sich weitere Angebote zur Verstetigung von Behandlungserfolg und Lebensstiländerung anschließen.

Die in der Reha angeregte Psychotherapie verzögerte sich aus diversen, leider alltäglichen Gründen: Primat der somatischen Behandlung und beruflichen Klärung bei geringen Zeitressourcen in der Hausarztpraxis, Probleme der ambulanten Psychotherapieplatzsuche (z. B. fehlende Rückrufe auf Nachrichten auf dem Anrufbeantworter), mangelnde somatische Kenntnisse des zuerst aufgesuchten Psychotherapeuten, lange Wartezeiten auf einen regulären Psychotherapieplatz, Antidepressiva-Nebenwirkungen bei insgesamt problematischer Adhärenz. Während der mehrmonatigen Wartezeit kam es dann zu einer Zunahme der Depression und der herzphobisch ausgestalteten Angstsymptomatik mit agoraphobischem Auslösungsmuster und ausgeprägter Somatisierung inkl. wiederholten Vorstellungen auf der Notaufnahme. Der mehrfache Infarktausschluss und der einmalige Kontakt mit dem niedergelassenen Kardiologen führte weder zur ausreichenden Angstreduktion noch zur Umsetzung der empfohlenen Verhaltensänderungen.

\section{Merke}

Im Zuge einer Depression kann der Patient die empfohlenen Lebensstiländerungen nicht ausreichend umsetzen und hält sich ggf. auch nicht an seine Medikamentenverordnungen vom Kardiologen.

Erst nach dem 5. Aufenthalt auf der Notaufnahme konnte mit der Einschaltung des psychosomatischen Konsiliarius und der stationären internistisch-psychosomatischen Behandlung (die leider nur an ausgewählten Kliniken möglich und auch dort nicht immer so kurzfristig verfügbar ist) 
eine Wende eingeleitet werden. Der relativ günstige Behandlungsverlauf konnte sicher auf die vorangegangenen, insgesamt positiv erlebten Psychologen- bzw. Psychotherapeutenkontakte aufbauen. Zugleich stellte er aber, wie oft bei Patienten in ähnlicher Situation, hohe Anforderungen an die interaktionelle Kompetenz des gesamten Stationsteams und eine enge interdisziplinäre und interprofessionelle Kooperation. Auch mit der erreichten Verbesserung bleibt zur Festigung und Weiterentwicklung des psychotherapeutischen Erfolgs, für den Wiedereinstieg in das Berufsleben, die Neugestaltung der partnerschaftlichen Situation und die Verstetigung sekundärpräventiver Verhaltensweisen ein längerfristiger, koordinierter Behandlungsbedarf bestehen. Hierfür ist eine regelmäßige Kommunikation aller Behandler bzw. - bei gegebenen Rahmenbedingungen - ggf. eine „Blended Collaborative Care“ mit Beachtung sowohl der psychischen als auch der verhaltensabhängigen somatischen Krankheitsanteile sinnvoll.

\section{Interessenkonflikt}

Autorenhonorare vom Verlag Hogrefe Huber für die deutsche Fassung der Hospital Anxiety and Depression Scale. Vortragshonorare der Firmen Heel, Novartis und Servier. Variable Bezüge seitens des Arbeitgebers für psychokardiologische Behandlungen von Privatpatienten. Führende Positionen in psychosomatischen Fachgesellschaften.

\section{Autorinnen/Autoren}

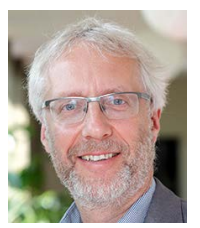

\section{Christoph Herrmann-Lingen}

Prof. Dr. med. Internist, Facharzt und Universitätsprofessor für Psychosomatische Medizin und Psychotherapie an der Universitätsmedizin Göttingen mit psychokardiologischem Forschungsschwerpunkt. Er ist Vorsitzender des Deutschen Kollegiums für Psychosomatische Medizin, Präsidiumsmitglied der Arbeitsgemeinschaft Wissenschaftlicher Medizinischer Fachgesellschaften und Past President der American Psychosomatic Society.

\section{Korrespondenzadresse}

Univ.-Prof. Dr. med. Christoph Herrmann-Lingen Klinik für Psychosomatische Medizin und Psychotherapie Georg-August-Universität Universitätsmedizin Göttingen

Von-Siebold-Straße 5

37099 Göttingen

cherrma@gwdg.de

\section{Wissenschaftlich verantwortlich gemäß Zertifizierungsbestimmungen}

Wissenschaftlich verantwortlich gemäß Zertifizierungsbestimmungen für diesen Beitrag ist Prof. Dr. med. Christoph Herrmann-Lingen, Göttingen.

\section{Erstveröffentlichung}

Dieser Beitrag wurde erstveröffentlicht in: PSYCH up2date 2019; 13: 59-74.

Literatur

[1] Joachim H. Papyros Ebers. Das älteste Buch über Heilkunde. Aus dem Aegyptischen zum ersten Mal vollständig übersetzt. Band 2. Berlin: Reimer; 1890

[2] Piepoli MF, Hoes AW, Agewall S et al. 2016 European Guidelines on cardiovascular disease prevention in clinical practice: The Sixth Joint Task Force of the European Society of Cardiology and Other Societies on Cardiovascular Disease Prevention in Clinical Practice (constituted by representatives of 10 societies and by invited experts). Developed with the special contribution of the European Association for Cardiovascular Prevention \& Rehabilitation (EACPR). Eur Heart J 2016; 37: 2315-2381

[3] Lichtman JH, Froelicher ESBlumenthal et al. Depression as a risk factor for poor prognosis among patients with acute coronary syndrome: Systematic review and recommendations: A scientific statement from the American Heart Asociation. Circulation 2014; 129: 1350-1369

[4] Bundesärztekammer (BÄK), Kassenärztliche Bundesvereinigung (KBV), Arbeitsgemeinschaft der Wissenschaftlichen Medizinischen Fachgesellschaften (AWMF). Nationale Versorgungs Leitlinie Chronische KHK - Langfassung, 4. Auflage 2016. Im Internet: https://www.leitlinien.de/mdb/ downloads/nvl/khk/khk-4aufl-vers1-kurz.pdf Stand: 03.02.2018

[5] Albus C, Waller C, Fritzsche $\mathrm{K}$ et al. Bedeutung von psychosozialen Faktoren in der Kardiologie. Update 2018. Kardiologe 2018; 12: 312-331

[6] Ponikowski P, Voors AA, Anker SD et al. 2016; ESC Guidelines for the diagnosis and treatment of acute and chronic heart failure. The Task force for the diagnosis and treatment of acute and chronic heart failure of the European Society of Cardiology (ESC). Developed with the special contribution of the Heart Failure Association (HFA) of the ESC. Eur J Heart Fail 2016: 2315-2381

[7] Braunschweig F, Boriani G, Bauer A et al. Management of patients receiving implantable cardiac defibrillator shocks: recommendations for acute and long-term patient management. Europace 2010; 12: 1673-1690

[8] Ghadri JR, Wittstein IS, Prasad A et al. International Expert Consensus Document on Takotsubo Syndrome (Part I): Clinical characteristics, diagnostic criteria, and pathophysiology. Eur Heart J 2018; 39: 2032-2046

[9] Albus C. Risikoverhalten. In Herrmann-Lingen C, Albus C, Titscher G. Psychokardiologie - ein Praxisleitfaden für Ärzte und Psychologen. Köln: Deutscher Ärzte-Verlag. 2014: 78-86 
[10] Herrmann-Lingen C, al'Absi M. Exploring the association of hypertension with risk for depression: Evidence for tamed neurobehavioral arousal versus central emotional dysregulation. Psychosom Med 2018; 80: 504-507

[11] Persu A, Petit G, Georges C et al. Hypertension, a posttraumatic stress disorder? Time to widen our perspective. Hypertension 2018; 71: 811-812

[12] Mann SJ. Psychosomatic research in hypertension: the lack of impact of decades of research and new directions to consider. J Clin Hypertens (Greenwich) 2012; 14: 657-664

[13] Dantzer R. Cytokine, sickness behavior, and depression. Immunol Allergy Clin North Am 2009; 29: 247-264

[14] Spitzer C, Meyer T, Herrmann-Lingen C. Komplexe Traumatisierungen und körperliche Gesundheit. Zur Assoziation von Kindesmisshandlungen und koronarer Herzkrankheit. Psychotherapeut 2016; 61: 191-196

[15] Waller C. (Trans-)Generationale Weitergabe früher Traumatisierung auf das kardiovaskuläre System. Psychotherapeut 2017; 62: 507-512

[16] Herrmann-Lingen C. Biopsychosoziale Faktoren in Genese und Manifestation der Koronaren Herzkrankheit. Z Psychosom Med Psychother 2000; 46: 315-330

[17] Meyer T, Herrmann-Lingen C. Natriuretic peptides in anxiety and panic disorder. Vitam Horm 2017; 103: 131-146

[18] Fangauf SV, Belnap BH, Meyer T et al. Associations of NTproBNP and parameters of mental health in depressed coronary artery disease patients. Psychoneuroendocrinology 2018; 96: 188-194

[19] Lespérance F, Frasure-Smith N, Koszycki D et al. Effects of citalopram and interpersonal psychotherapy on depression in patients with coronary artery disease: the Canadian Cardiac Randomized Evaluation of Antidepressant and Psychotherapy Efficacy (CREATE) trial. JAMA 2007; 297: 367-379

[20] Berkman LF, Blumenthal J, Burg M et al. Effects of treating depression and low perceived social support on clinical events after myocardial infarction: the Enhancing Recovery in Coronary Heart Disease Patients (ENRICHD) Randomized Trial. JAMA 2003; 289: 3106-3116

[21] Herrmann-Lingen C, Beutel ME, Bosbach A et al. A stepwise psychotherapy intervention for reducing risk in coronary artery disease (SPIRR-CAD): Results of an observer-blinded, multicenter, randomized trial in depressed patients with coronary artery disease. Psychosom Med 2016; 78: 704-715

[22] Jeyanantham K, Kotecha D, Thanki D. Effects of cognitive behavioural therapy for depression in heart failure patients: a systematic review and meta-analysis. Heart Fail Rev 2017; 22: 731-741

[23] DGPPN BÄKKBV et al.S3-Leitlinie/Nationale VersorgungsLeitlinie Unipolare Depression - Langfassung, 2. Auflage. Version 5. 2015. Im Internet: https://www.awmf.org/ uploads/tx_szleitlinien/nvl-005I_S3_Unipolare_ Depression_2017-05.pdf Stand: 14.08.2018

[24] Glassman AH, O'Connor CM, Califf RM et al. Sertraline treatment of major depression in patients with acute MI or unstable angina. JAMA 2002; 288: 701-709

[25] Kim JM, Bae KY, Stewart R et al. Escitalopram treatment for depressive disorder following acute coronary syndrome: a 24-week double-blind, placebo-controlled trial. J Clin Psychiatry 2015; 76: 62-68
[26] O'Connor CM, Jiang W, Kuchibhatla M et al. Safety and efficacy of sertraline for depression in patients with heart failure: results of the SADHART-CHF (Sertraline Against Depression and Heart Disease in Chronic Heart Failure) trial. J Am Coll Cardiol 2010; 56: 692-699

[27] Angermann CE, Gelbrich G, Störk S et al. Effect of escitalopram on all-cause mortality and hospitalization in patients with heart failure and depression: The MOOD-HF Randomized Clinical Trial. JAMA 2016; 315: 2683-2693

[28] Kim JM, Stewart R, Lee YS et al. Effect of escitalopram vs. placebo treatment for depression on long-term cardiac outcomes in patients with acute coronary syndrome: A randomized clinical trial. JAMA 2018; 320: 350-358

[29] Olewinski M, Paschke AM, Mohebbi A et al. An integrative psychosomatic Approach to the Treatment of Patients with cardiovascular Diseases: Concepts and Experiences of a dedicated Psychocardiology Ward at the University of Göttingen Medical Center. In Baune BT, Tully PJ. (eds.). Cardiovascular Diseases and Depression - Treatment and Prevention in Psychocardiology. Cham: Springer; Switzerland: 2016: 493-507

[30] Tully PJ, Baumeister H. Collaborative care for comorbid depression and coronary heart disease: a systematic review and meta-analysis of randomized controlled trials. BMJ Open 2015; 5: e009128

[31] Meyer T, Belnap BH, Herrmann-Lingen C et al. Benefits of collaborative care for post-CABG depression are not related to adjustments in antidepressant pharmacotherapy. J Psychosom Res 2014; 76: 28-33

[32] Katon W], Lin EH, Von Korff M et al. Collaborative care for patients with depression and chronic illnesses. N Engl J Med 2010; 363: 2611-2620

[33] Rossom RC, Solberg LI, Magnan S et al. Impact of a national collaborative care initiative for patients with depression and diabetes or cardiovascular disease. Gen Hosp Psychiatry 2017; 44: 77-85

[34] Bosselmann L, Herbeck Belnap B, Fangauf SV et al. Durchführbarkeit und Wirksamkeit eines kollaborativen Behandlungsansatzes in der Sekundärprävention der Koronaren Herzkrankheit. Z Psychosom Med Psychother 2018; 64: 44-45 (abstract)

[35] Kohrs M, Die Narzisstische Persönlichkeitsstörung im Spannungsfeld unterschiedlicher Theorien und Behandlungsansätze.In Boll-Klatt A, Kohrs M. Praxis der psychodynamischen Psychotherapie. 2. Aufl. Stuttgart: Schattauer; 2018: 516-542

Bibliografie

DOI https://doi.org/10.1055/a-0870-1420

Psychother Psych Med 2019; 69: 237-252

(c) Georg Thieme Verlag KG Stuttgart · New York ISSN 0937-2032 


\section{Punkte sammeln auf CME.thiemeode}

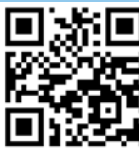

Diese Fortbildungseinheit ist 12 Monate online für die Teilnahme verfügbar. Sollten Sie Fragen zur Online-Teilnahme haben, finden Sie unter cme.thieme.de/hilfe eine ausführliche Anleitung. Wir wünschen viel Erfolg beim Beantworten der Fragen!

Unter https://eref.thieme.de/CXCSF88 oder über den QR-Code kommen Sie direkt zum Artikel zur Startseite des Wissenstests.

VNR 2760512019156640813

\section{Frage 1}

Was ist kein psychosozialer kardiologischer Risikofaktor gemäß der europäischen Leitlinie zur Prävention von Herz-Kreislauf-Erkrankungen?

A Stress im Beruf und Familienleben

B Depression

C Typ-A-Verhaltensmuster

D niedriger sozioökonomischer Status

E Angst

\section{Frage 2}

Welche Aussage trifft zu?

A Die morphologische Schwere einer Herzerkrankung korreliert höchstens schwach mit der subjektiv empfundenen Beeinträchtigung.

B Entzündungsprozesse zeigen keine Wechselwirkung mit psychischen Faktoren.

C Psychische Störungen mit Krankheitswert nach einer Herzerkrankung betreffen die Mehrheit der Erkrankten.

D Die regelmäßige Medikamenteneinnahme ist förderlich für den psychischen Umgang der Patienten mit ihrer Herzerkrankung.

E Die Einnahme von Sacubitril hemmt den Abbau natriuretischer Peptide und beeinträchtigt dadurch das psychische Befinden.

\section{Frage 3}

Wovon hängt die gesundheitsbezogene Lebensqualität von Patienten mit Herzerkrankungen stark ab?
A vom systolischen Blutdruck
B vom Ausmaß der psychosozialen Belastung
C vom Zeitpunkt seit der Diagnose
D vom CRP-Wert
E vom Alter des Patienten

\section{Frage 4}

Worauf ist bei der Diagnose einer psychischen Komorbidität bei Herzpatienten zu achten?

A Sie sollte in Anwesenheit eines Familienangehörigen erfolgen.

B Sie kann erst nach einer intensiven laboranalytischen Untersuchung gestellt werden.

C Die Überlappung von psychischen und kardialen Symptomen kann zu Fehleinschätzungen führen.

D Herzpatienten neigen mehr als andere Patienten zu Übertreibungen ihrer Symptome.

E Supraventrikuläre Bradykardien können vorschnell als Panikstörung gedeutet werden.

\section{Frage 5}

Was ist ein wichtiger Bestandteil der psychosomatischen Therapie bei Herzpatienten?

A Kardiologische Vorstellungen im vierteljährlichen Rhythmus

B Psychologische Interventionen z. B. mit Stressbewältigungstrainings

C Eine antidepressive Therapie mit Amitriptylin

D Eine symptomzentrierte Kommunikation

E Eine 4-wöchige interpersonelle Psychotherapie

\section{Frage 6}

Für welche Therapie liegt bei depressiven Patienten mit Herzinsuffizienz die stärkste Evidenz hinsichtlich der Besserung von depressiven Symptomen und Lebensqualität vor?

A selektive Serotonin-Wiederaufnahmehemmer

B körperliches Training

C analytische Psychotherapie

D kognitive Verhaltenstherapie

E Collaborative Care 


\section{Punkte sammeln auf CME.thieme.de}

\section{Fortsetzung ...}

\section{Frage 7}

Welches der folgenden Antidepressiva sollte bei Patienten mit KHK oder Herzinsuffizienz vermieden werden?
A Amitriptylin
B Citalopram
C Escitalopram
D Mirtazapin
E Sertralin

\section{Frage 8}

Für welches Antidepressivum liegt bei depressiven KHKPatienten die beste Evidenz für einen möglichen prognostischen Nutzen vor?
A Amitriptylin
B Citalopram
C Escitalopram
D Mirtazapin
E Sertralin

\section{Frage 9}

Welcher EKG-Parameter sollte bei Einsatz einer psychopharmakologischen Behandlung von Herzpatienten aus Sicherheitsgründen vor allem kontrolliert werden?
A Herzfrequenz
B Herzfrequenzvariabilität (z. B. SDNN)
C ST-Streckensenkung
D QTc-Intervall
E Kammerkomplexbreite

\section{Frage 10}

Was gilt für die Langzeitbetreuung von Patienten mit chronischer KHK?

A Neben jährlichen kardiologischen Kontrollen sind bei asymptomatischen Patienten keine weiteren Maßnahmen erforderlich.

B In der Regel führt der Schreck über die Diagnosestellung zu einer konsequenten Umstellung des Lebensstils.

C Wegen ihrer thrombozytenaggregationshemmenden und depressionsprotektiven Wirkung sollten selektive Serotonin-Wiederaufnahmehemmer regelmäßig verordnet werden.

D Wegen der hohen Relevanz psychosozialer Faktoren sollte jedem KHK-Patienten eine Verhaltenstherapie angeboten werden.

E Die aktuelle Depressionsleitlinie empfiehlt eine Psychotherapie für depressive KHK-Patienten. 


\title{
Psychocardiology - Current Guidelines and Clinical Reality
}

\begin{abstract}
In the last decades, psychocardiological knowledge has contributed a lot to the understanding of psychosocial factors involved in the pathogenesis, course, and treatment of cardiovascular diseases. Therefore, psychological, social, and behavioral factors should be detected and addressed early on in order to prevent cardiovascular disease and/or its progression. On the other hand, cardiovascular disease is an important stressor which can lead to psychological comorbidities and thus deteriorate prognosis and quality of life. During therapy a clear, consistent, and empathic communication between professionals and patients is of importance. Up to now there is not much evidence as to the effectiveness of psychotherapeutic interventions or antidepressant medication for cardiac patients with mental comorbidities. Beyond psychosomatic basic care, combined with physical activity, severely ill patients may require multimodal inpatient care in specialized psychosomatic or psychocardiological units and continuous support with lifestyle change.
\end{abstract}

Schlüsselwörter

Diagnose, Psychosomatik, Psychotherapie, pharmakologische

Behandlung, psychologische Komorbidität

Key words

Diagnosis, psychosomatics, psychotherapy, pharmacological treatment, psychological comorbidity 